\title{
A influência do estado nutricional e da ingestão alimentar na aprendizagem escolar
}

\section{The influence of nutritional status and food intake in academic achievement}

\author{
1 Anaflor Moreira \\ 1 Nicolas Charon Moeda Benedetti \\ 2 Margareth Lopes Galvão Saron \\ 2 Alden dos Santos Neves \\ 2 Elton Bicalho de Souza elton bicalho@ig.com.br
}

1 Discente do curso de Nutrição do Centro Universitário de Volta Redonda, UniFOA.

2 Docente do curso de Nutrição do Centro Universitário de Volta Redonda, UniFOA.

\section{RESUMO}

Segundo a literatura, alguns nutrientes possuem efeito positivo no funcionamento do cérebro, e alguns estão relacionados à memória. Em contrapartida, uma alimentação desequilibrada exerce efeitos não desejados sobre a cognição, gerando declínio no desempenho. 0 objetivo do presente estudo foi verificar possíveis associações entre estado nutricional e ingestão alimentar com o rendimento escolar em alunos do $6^{\circ}$ ao $9^{\circ}$ ano de uma escola de Volta Redonda, RJ. Trata-se de um estudo transversal, no qual foi aplicado um questionário, bem como o levantamento de dados nas fichas de registro. 0 estado nutricional foi mensurado através do índice de massa corporal. A avaliação do consumo alimentar foi aferida por meio de um questionário de frequência alimentar. Foi avaliado um total de 90 alunos, com maioria do sexo feminino e com maior prevalência de eutrofia. A maior parte dos participantes relatou realizar mais de 4 refeições por dia. Constatou-se ingestão inadequada de ômega 3 , zinco, ferro, sódio e açúcar pela maioria dos participantes. No presente estudo não foi encontrada associação estatística significativa entre estado nutricional e consumo alimentar com o desempenho escolar da amostra, porém observou-se alta prevalência na deficiência do consumo de ômega 3 , zinco e ferro e excesso no consumo de sódio.

\section{PALAVRAS-CHAVE}

Estado nutricional; nutrição do adolescente; saúde escolar.

\begin{abstract}
According to the literature, some nutrients have a positive effect on brain function, and some are related to memory. In contrast, a poor diet exerts undesirable effects on cognition, resulting in performance decline. The purpose of this study was to investigate possible associations between nutritional status and food intake with academic achievement in the 6th to 9th grade students enrolled in a school in Volta Redonda, RJ. It was a transversal study, where a questionnaire was applied and data was collected from registration forms. Nutritional status was measured by body mass index. The evaluation of dietary intake was measured using a food frequency questionnaire. $A$ total of 90 students were evaluated, most females and with greater prevalence of average weight and most participants reported consuming more than 4 meals a day. It was verified inadequate intake of omega 3 , zinc, iron, sodium and sugar by most participants. In this study there was no significant statistical association linking nutritional status and food intake to school performance of the sample. However, there was high prevalence of deficiency in omega 3, zinc and iron consumption, and excessive consumption of sodium.
\end{abstract}

\section{KEYWORDS}

Nutritional status; adolescent nutrition; school health.

\section{Como você deve citar?}

SOUZA, Elton Bicalho de et al.. A influência do estado nutricional e da ingestão alimentar na aprendizagem escolar. Cadernos UniFOA, Volta Redonda, n. 29, p. 105-113, dez. 2015. 


\section{INTRODUÇÃO}

Os adolescentes no Brasil representam cerca de $25 \%$ da população, o que equivale a uma fração relevante em nosso país. Nessa fase, ocorrem diversas mudanças nos âmbitos físicos, sociais e comportamentais desses indivíduos, deixando esses jovens em maior risco de possíveis distúrbios nutricionais, pois afetam diretamente seus hábitos alimentares e estilo de vida (BRASIL, 2007). Geralmente os adolescentes aderem a padrões dietéticos por influência da família, dos amigos e da mídia. Por um lado, existe a valorização da sociedade pelo corpo magro, o que pode levar a transtornos alimentares, como anorexia e bulimia. Em contrapartida, há um aumento de casos de sobrepeso e obesidade entre os adolescentes no Brasil.

Além disso, as carências nutricionais nessa faixa etária, como a desnutrição e a anemia ferropriva, embora menos prevalentes do que a obesidade, são problemas que necessitam de atenção, devido à repercussão que causam à saúde (ACCIOLY, 2009). Existe uma demanda no aporte energético e de micronutrientes em função das alterações fisiológicas ocorridas na adolescência e, por isso, uma alimentação desequilibrada pode ocasionar atraso no crescimento e desenvolvimento (PRIORE, 2010).

A literatura mostra que diversos nutrientes exercem um efeito positivo em relação ao cérebro, sendo que alguns são relacionados especificamente à memória. Os alimentos que contêm ácidos graxos poli-insaturados ômega 3 melhoram a resposta das sinapses e são essenciais para o funcionamento do cérebro. (GÓMEZ-PINILLA, 2008). 0 ferro é encontrado em maior concentração em áreas nobres cerebrais, por ser necessário à síntese de enzimas envolvidas no processo de mielinização das fibras nervosas e na síntese de neurotransmissores. Sua deficiência reflete-se negativamente no desenvolvimento mental, levando à redução da capacidade de concentração e do aprendizado. 0 zinco atua na atividade neuronal $\mathrm{e}$ na memória e sua deficiência pode afetar as funções desempenhadas pelo sistema nervoso (COZZOLINO, 2009). Em contrapartida, estudos mostram que uma alimentação rica em açúcar, sódio, gorduras saturadas e gorduras trans têm efeitos negativos sobre a cognição mental. Uma dieta com essas características leva a um declínio no desempenho cognitivo e redução de plasticidade sináptica no hipocampo, sugerindo que esse tipo de alimentação tem um impacto direto sobre os neurônios (GÓMEZ-PINILLA, 2008).

Segundo Pontes (2010), uma dieta balanceada é fundamental para o crescimento e desenvolvimento intelectual, pois melhora o nível educacional e reduz transtornos de aprendizado causados por deficiências nutricionais. Entretanto, dados de estudos sobre a ingestão alimentar nacional de crianças e adolescentes indicaram que grande parte das dietas não atinge as recomendações nacionais para esse grupo populacional (MAHAN; ESCOTT-STUMP; RAYMOND 2013).

Diante do exposto, o objetivo do presente trabalho foi verificar possíveis associações entre estado nutricional e ingestão alimentar com o rendimento escolar em alunos do $6^{\circ}$ ao $9^{\circ}$ ano, matriculados em uma escola do município de Volta Redonda, RJ.

O estudo justifica-se por ser a alimentação de fundamental importância no desenvolvimento intelectual do adolescente. Glewwe, Jacoby e King (2001) afirmam que a interação entre nutrição e aprendizado tem crescido, apresentando nos últimos anos um considerável aumento de estudos que expõem argumentos convincentes, no que se refere ao impacto da nutrição no desempenho dos escolares.

\section{MÉTODOS E TÉCNICAS}

Trata-se de um estudo quantitativo, de desenho transversal, que foi realizado em uma escola da rede estadual de Volta Redonda, interior do estado do Rio de Janeiro. Os participantes foram captados 
durante a reunião de pais e professores e convidados a participarem da pesquisa. A amostra foi composta de alunos do $6^{\circ}$ ao $9^{\circ}$ anos que deveriam atender aos seguintes critérios de inclusão: aceite em participar de forma espontânea, permissão formal do responsável para participação da criança com assinatura do termo de consentimento livre e esclarecido. Foram considerados critérios de exclusão alunos com alguma deficiência física ou mental e recusa em participar da pesquisa, ainda que com consentimento do responsável.

A caracterização da amostra foi realizada por meio da aplicação de um questionário com os participantes e levantamento de dados nas fichas de registro e informações fornecidas por professores ou orientadores educacionais da escola. 0 estado nutricional foi mensurado através do índice de massa corporal (IMC). Para verificação do IMC dos participantes, foi levado em consideração o peso (kg) e a estatura $\left(\mathrm{m}^{2}\right)$, aferidos após o preenchimento do questionário. O diagnóstico do estado nutricional através do IMC foi de acordo com as curvas de IMC para idade e sexo, da Organização Mundial de Saúde (WHO, 2006).

A avaliação do consumo alimentar foi aferida por meio de um questionário de frequência alimen$\operatorname{tar}$ (QFA), aplicado diretamente com os estudantes. Esse QFA foi adaptado para mensurar o consumo das principais fontes de nutrientes com efeitos positivos e negativos para o cérebro, segundo revisão da literatura realizada. A análise quantitativa dos nutrientes foi realizada com o auxílio do programa AVANUTRI ${ }^{\circledR}$ versão Revolution 4.0.

Em linhas gerais, as variáveis foram analisadas, segundo procedimentos clássicos de média e desvio padrão, com auxílio do programa Excel for Windows ${ }^{\oplus}$. Para verificar possíveis associações entre estado nutricional, consumo e desempenho escolar, foi realizado o teste estatístico do qui-quadrado, com auxílio do programa S.P.S.S, levando em consideração o nível de significância de $P>0,05$.

O trabalho foi aprovado pelo Comitê de Ética em Pesquisa do Centro Universitário de Volta Redonda, sob o número do CAAE 37610314.4.0000.5237.

\section{RESULTADOS E DISCUSSÃO}

Foi avaliado um total de 90 alunos, com maioria $(n=52)$ do sexo feminino e com média de idade de 12,4 $( \pm 1,58)$ anos. A avaliação dos dados antropométricos demonstrou uma média de peso de 48,4 $( \pm 11,2) \mathrm{kg}$, altura de $160( \pm 0,1) \mathrm{cm}$ e IMC de $19,3( \pm 3,3) \mathrm{kg} / \mathrm{m}^{2}$, conforme ilustra a tabela 1 . Quando estratificados por sexo, nota-se que as meninas possuíam maiores valores de peso, estatura e IMC, conforme apresenta a tabela 2.

A distribuição da amostra com relação ao estado nutricional, de acordo com as curvas de IMC para idade e sexo da Organização Mundial de Saúde (WHO, 2006), está ilustrada ao gráfico 1.

Tabela 1 - Variáveis antropométricas dos alunos participantes do estudo

\begin{tabular}{cccc}
\hline Variável & Média & Desvio Padrão & Mínimo/Máximo \\
\hline Peso $(\mathrm{kg})$ & 48,4 & $+11,2$ & $25,6-77,7$ \\
Estatura $(\mathrm{cm})$ & 160 & $+0,1$ & $130-182$ \\
$\mathrm{IMC}\left(\mathrm{kg} / \mathrm{m}^{2}\right)$ & 19,3 & $+3,3$ & $14,7-30,3$ \\
\hline
\end{tabular}

Fonte: dos autores. 
Tabela 2 - Variáveis antropométricas estratificadas por sexo

\begin{tabular}{ccccccc}
\hline \multirow{2}{*}{ Variável } & \multicolumn{3}{c}{ Média } & \multicolumn{2}{c}{ Desvio Padrão } & \multicolumn{2}{c}{ Mínimo/Máximo } \\
\cline { 2 - 7 } & $\mathbf{M}$ & $\mathbf{F}$ & $\mathbf{M}$ & $\mathbf{F}$ & $\mathbf{M}$ & $\mathbf{F}$ \\
\hline Peso $(\mathrm{kg})$ & 45,8 & 50,8 & $+3,39$ & $+8,69$ & $25,6-73,6$ & $29,3-77,7$ \\
Estatura $(\mathrm{cm})$ & 156 & 158 & $+0,07$ & $+0,04$ & $130-182$ & $140-169$ \\
IMC $\left(\mathrm{kg} / \mathrm{m}^{2}\right)$ & 18,4 & 20,1 & $+0,05$ & $+2,11$ & $14,7-26,9$ & $14,7-30,3$ \\
\hline
\end{tabular}

Legenda: $M=$ masculino; $F=$ feminino Fonte: dos autores.

Gráfıco 1 - Distribuição do estado nutricional dos participantes estratificados por sexo

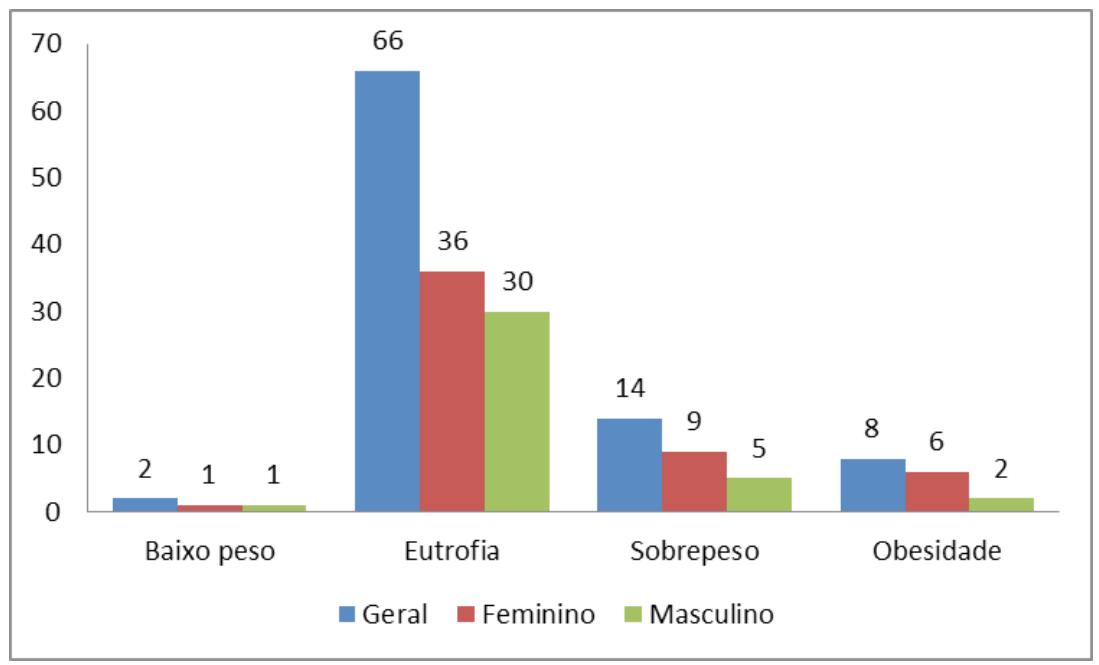

Fonte: dos autores.

O presente estudo verificou maior prevalência de eutrofia (73\%) entre os participantes. Esse achado é semelhante ao estudo realizado por Neto et al. (2008), na cidade de São Mateus do Sul, Paraná, com adolescentes entre 12 e 16 anos. Os autores descrevem que $84 \%$ dos alunos estavam eutróficos. Já no estudo realizado por Garcia, Gambardella e Frutuoso (2003), na cidade de São Paulo, verificou-se valores similares ao presente estudo, com prevalência de $2 \%$ de baixo peso, $78 \%$ de eutrofia, $12 \%$ de sobrepeso e $8 \%$ de obesidade.

A maior parte de eutrofia dos participantes é contrária a muitos estudos que apresentam dados antropométricos de escolares no Brasil. Segundo Da Veiga, Da Cunha e Sichieri (2004), as prevalências de excesso de peso têm aumentado consideravelmente nos países em desenvolvimento e, no Brasil, a comparação entre o Estudo Nacional de Despesa Familiar (ENDEF), em 1974-1975, e a Pesquisa sobre Padrões de Vida (PPV), em 1996-1997, indicam que, em apenas duas décadas, o excesso de peso é mais do que o dobro entre as meninas (de $5,8 \%$ para $15,3 \%$ ) e mais do que o triplo entre os meninos (de 2,6\% para 11,8\%). Dados obtidos a partir da Pesquisa Nacional de Orçamentos Familiares (POF), realizada em 2002-2003, apontam que o excesso de peso atinge $17,9 \%$ e $15,4 \%$ dos adolescentes brasileiros de sexo masculino e feminino, respectivamente. Além disso, segundo a POF, 2,3\% dos jovens apresentavam obesidade (BRASIL, 2006). Em 2009, a Pesquisa Nacional de Saúde do Escolar (PENSE) apontou aumento na prevalência do excesso de peso (23\%) e obesidade de $(7,3 \%)$, sempre maiores nos indivíduos do sexo masculino (ARAÚJO et al., 2010) 
Quando o estado nutricional é avaliado estratificado por sexo, percebe-se maior prevalência de sobrepeso e obesidade nas meninas. Diversos estudos detectaram predominância de excesso de peso entre adolescentes do sexo feminino. Em 1989, a Pesquisa Nacional de Saúde e Nutrição (PNSN) constatou aumento na prevalência de sobrepeso entre adolescentes, principalmente do sexo feminino (SANTOS et al., 2005).

Com relação aos hábitos alimentares, a maioria $(n=49)$ relatou realizar de 4 a 5 refeições diárias, conforme apresenta o gráfico 2.

Gráfico 2 - Número de refeições realizadas por dia pelos alunos participantes da pesquisa

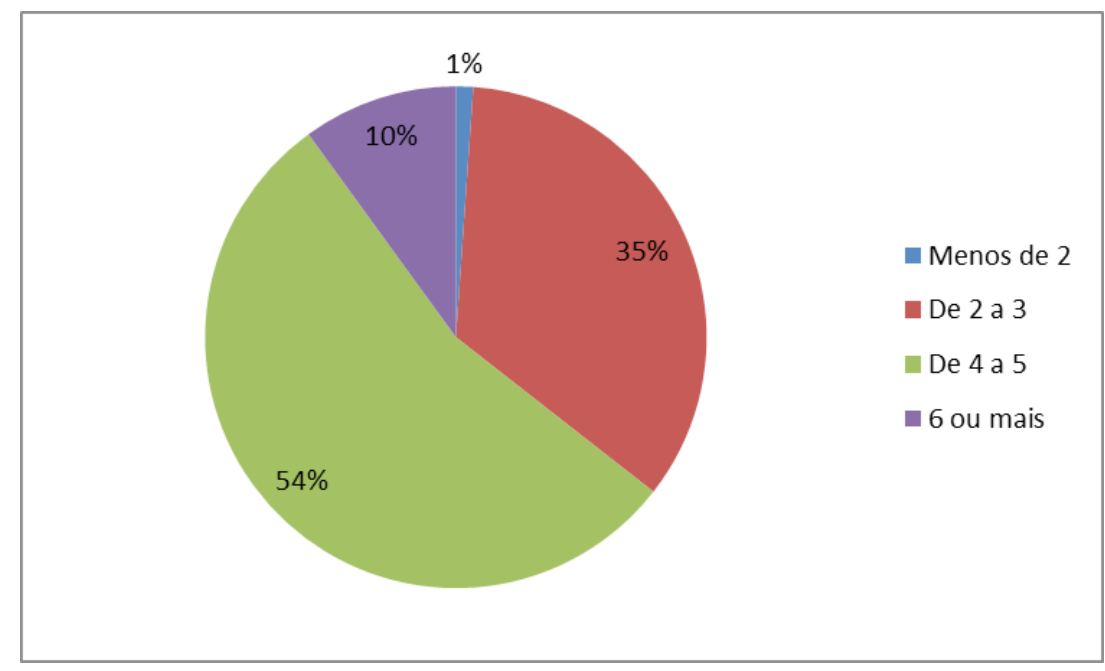

Fonte: dos autores.

O achado vai ao encontro da pesquisa de Dalla Costa, Cordoni Júnior e Matsuo (2007), realizado na cidade de Toledo, Paraná, que encontra um valor semelhante de $55,6 \%$ de predominância de consumo de 4 ou mais refeições ao dia. $\mathrm{O}$ maior número de refeições diárias apresenta-se como fator de proteção para o excesso de peso entre adolescentes, independente da idade e sexo (REZENDE et al., 2009). Mahan e Escott-Stump (2013) destacam que o ideal seria o consumo de mais de 6 refeições ao dia, pois refeições realizadas em menor quantidade e maior frequência garantem um menor risco de desenvolvimento de obesidade. Na presente pesquisa apenas $10 \%(n=9)$ dos alunos relataram realizar mais de 6 refeições. Para $34 \%(n=31)$ dos estudantes, o número de refeições diárias ficou entre 2 a 3 . 0 ato de pular refeições é um hábito comum entre adolescentes, 0 que pode contribuir para a dificuldade de alcançar as necessidades nutricionais recomendadas (LEAL et al., 2010). Além disso, segundo Mahan, Escott-Stump e Raymond (2013), a omissão de refeições pode levar a uma diminuição de nutrientes essenciais para que os adolescentes mantenham um estado nutricional saudável. Constatou-se que $63,3 \%(n=57)$ dos alunos realizam as refeições em casa e na escola e o restante, $36,6 \%(n=33)$, somente em casa. Ressalta-se então a importância da merenda escolar na promoção da saúde dos escolares. Sabe-se que os hábitos alimentares são influenciados pelo meio e que a escola é um local em que as crianças e adolescentes passam grande parte do seu tempo, influenciando na formação de opiniões e conceitos. Portanto, deve-se buscar estabelecer ações que promovam a saúde alimentar dos estudantes (CAMPOS; ZUANON, 2010). Em referência ao desempenho escolar, de acordo com o questionário aplicado aos estudantes, $40 \%(n=36)$ deles já haviam sido reprovados, sendo que a maioria $(n=21)$ repetiu uma única vez. Também foram analisados o número de recuperações, constatando-se que $44,4 \%(n=40)$ dos alunos relataram já ter ficado com dependência em determinada matéria, sendo que grande parte $(n=23)$ havia ficado apenas uma única 
vez. A maioria dos participantes $(n=54)$ relatou apresentar dificuldade em uma matéria específica, em que a mais citada foi matemática, com $66,6 \%$.

A análise de adequação da ingestão dos nutrientes foi realizada de acordo com os parâmetros da Dietary Reference Intake - DRI (IOM, 2002; IOM, 2004; IOM, 2005; IOM, 2006). Na avaliação do questionário de consumo alimentar, constatou-se que $97,7 \%$ dos escolares têm ingestão insuficiente de ômega 3, zinco e ferro. Em relação ao sódio, 96,6\% têm ingestão acima do preconizado, ressaltando-se que não foi avaliado o sal adicionado aos alimentos. Em relação ao açúcar, houve baixa ingestão em $60 \%$ dos participantes.

Apesar da alta prevalência de inadequação no consumo de nutrientes, não foi encontrada associação estatística significativa entre as variáveis do presente estudo. Entretanto, estudos mostram que os nutrientes citados têm relação com o funcionamento cerebral e que sua deficiência pode levar a alterações em funções cognitivas.

O ferro é essencial para o desenvolvimento e funcionamento adequado do cérebro. A deficiência de ferro no início da vida está associada a níveis significativamente inferiores de cognição e desenvolvimento infantil comportamental, deterioração grave da função neuronal no hipocampo, falta de memória e capacidades de aprendizagem espacial (HAN; KIM, 2015). 0 estudo de Muñoz et al. (2011) demonstrou que a suplementação de ferro aumenta a plasticidade sináptica do cérebro por ativação do $\mathrm{N}$-metil-D-aspartato, um receptor associado com a função de memória, sugerindo que o nível de ferro no cérebro altera significativamente os percursos dos neurotransmissores e atividades neuronais.

O zinco está presente no cérebro através de ligação com proteínas e é importante para a estrutura e função dele. Há evidências que sugerem que a deficiência de zinco reduz os níveis de ômega 3 e ômega 6 de cadeia longa, prejudicando o metabolismo de ácidos graxos nos neurônios. Além disso, 0 zinco parece ser importante para a neurogênese, migração neuronal e sinaptogênese e sua deficiência poderia interferir com as neurotransmissões e com o desenvolvimento neurofisiológico. 0 zinco também está relacionado ao metabolismo de hormônios da tiroide e transporte de outros hormônios, o que poderia influenciar no sistema nervoso central (BHATNAGAR; TANEJA, 2001). Foi realizada uma série de diferentes estudos em macacos por Golub et al. $(1984 ; 1988 ; 1991 ; 1994 ; 1996)$, nos quais foram examinadas suas funções cognitivas em diferentes estágios de maturação sexual, após um período de privação de zinco durante a gravidez, a infância até a adolescência. 0 comportamento avaliado em diversos estágios de maturação sexual - infantil, juvenil e adolescência, mostraram uma hipoatividade geral e redução das respostas ao ambiente em animais na infância. Macacos na fase juvenil foram incapazes de repetir tarefas aprendidas anteriormente e tinham dificuldades em aprender novos problemas. Efeitos em funções cognitivas como aprendizado e memória foram mais acentuados em animais adolescentes privados de zinco.

De acordo com Bourre (2004), experimentos demonstraram que a deficiência de ômega 3 na dieta teve efeito sobre a estrutura e função do cérebro. Foi verificado que a deficiência altera o desenvolvimento cerebral, a composição de propriedades de neurônios, oligodendrócitos e astrócitos. 0 estudo de Bos et al. (2015), realizado com meninos entre 8 e 14 anos, na Holanda, registrou que a suplementação dietética de ômega 3 melhorou sintomas de desatenção em meninos com e sem o transtorno de déficit de atenção. Esses resultados sugerem que crianças com desenvolvimento normal também se beneficiam, mostrando a importância da ingestão de ômega 3.

O estudo de Berti et al. (2015), realizado com 52 indivíduos, verificaram a relação entre a ingestão de nutrientes com a função cognitiva e observaram associação negativa da ingestão de gorduras saturadas, gorduras trans e sódio com volume da massa cinzenta e atividade metabólica. Já o zinco 
obteve associação positiva com a massa cinzenta. Granholm et al. (2008), em um estudo com 344 ratos, demonstraram que gorduras saturadas e hidrogenadas suplementadas na forma de óleo de coco hidrogenado, adicionado de colesterol na dieta, aumentou as alterações de comportamento e morfologia cerebral na cobaias, quando comparadas a ratos que possuíam dieta com baixo colesterol e níveis iguais de gorduras insaturadas. Esses resultados demonstraram que o consumo de gorduras saturadas e hidrogenadas aumentou significativamente o número de erros da memória e nos marcadores microgliais na formação do hipocampo.

\section{CONCLUSÃO}

Segundo a literatura, o estado nutricional e o consumo de nutrientes exercem efeitos positivos ou negativos no desempenho escolar, muito por alteração das funções cognitivas. Diversos trabalhos realizados com humanos e animais mostraram a importância do ferro, zinco e ômega 3 no funcionamento cerebral, na atividade neuronal e na memória. Em contrapartida, estudos demonstram que a alta ingestão de sódio causa redução da massa cinzenta cerebral, do desempenho cognitivo e das respostas sinápticas no hipocampo, tendo relação direta com o funcionamento ineficiente dos neurônios.

No presente estudo não foi encontrada associação estatística significativa entre estado nutricional e consumo alimentar com o desempenho escolar da amostra, porém observou-se alta prevalência na deficiência do consumo de ômega 3, zinco e ferro e excesso no consumo de sódio. A inadequação desses nutrientes pode causar declínio em funções cognitivas e na capacidade de concentração.

A inadequação do consumo dos nutrientes detectada no presente estudo é preocupante, uma vez que sugere que a alimentação realizada pelos participantes não está suprindo as necessidades, podendo afetar o desempenho e a saúde desses indivíduos. Destaca-se então a necessidade da realização de mais estudos sobre o presente tema, para tentar minimizar possíveis problemas e traçar estratégias de educação nutricional para corrigir a deficiência na ingestão alimentar dessa população.

\section{AGRADECIMENTOS}

Os autores agradecem o fomento do Programa de Iniciação Científica do Centro Universitário de Volta Redonda - UniFOA.

\section{REFERÊNCIAS}

ACCIOLY, E.; SAUNDERS, C.; LACERDA, E. M. de A.. Nutrição em obstetrícia e pediatria. 2.ed. Rio de Janeiro: Cultura Médica, 2009. 649p.

ARAÚJJ, C. et al. Estado nutricional dos adolescentes e sua relação com variáveis sociodemográficas: Pesquisa Nacional de Saúde do Escolar (PeNSE), 2009. Ciência e Saúde Coletiva, v. 15, n. 2, p. 3077-3084, 2010.

BRASIL. Ministério da Saúde. Marco legal: saúde, um direito de adolescentes. Normas e Manuais Técnicos. Brasília, p. 60, 2007.

BERTI V. et al. Nutrient patterns and brain biomarkers of alzheimer's disease in cognitively normal individuals. The journal of nutrition, health \& aging. v. 19, n. 4, p. 413-423, 2015. 
BHATNAGAR, S.; TANEJA, S.. Zinc and cognitive development. British journal of nutrition, v. 85, n. 2, p. 139-145, 2001.

BOS, D. J. et al. Reduced Symptoms of Inattention after Dietary Omega-3 Fatty Acid Supplementation in Boys with and without Attention Deficit/Hyperactivity Disorder. Neuropsychopharmacology, 2015.

BOURRE, J. M. Roles of unsaturated fatty acids (especially omega- 3 fatty acids) in the brain at various ages and during ageing. The journal of nutrition, health \& aging, v. 8, n. 3, 2004.

CAMPOS, J. A. D. B.; ZUANON, A. C. C. Merenda escolar e promoção de saúde. Brazilian Dental Science, v. 7, n. 3, 2010.

COZZOLINO, S. M. F.. Biodisponibilidade de nutrientes. 3. ed. Barueri: Manole, 2009. 1172 p.

DA VEIGA, G. V.; DA CUNHA, A. S.; SICHIERI, R. Trends in overweight among adolescents living in the poorest and richest regions of Brazil. American Journal of Public Health, v. 94, n. 9, p. 1544-1548, 2004.

DALLA COSTA, M. C.; CORDONI JÚNIOR, L.; MATSUO, T. Hábito alimentar de escolares adolescentes de um município do oeste do Paraná. Revista de Nutrição, v. 20, n. 5, p. 461-471, 2007.

GARCIA, G. C. B.; GAMBARDELLA, A. M. D.; FRUTUOSO, M. F. P. Estado nutricional e consumo alimentar de adolescentes de um centro de juventude da cidade de São Paulo. Revista de Nutrição, v. 16, n. 1, p. 41-50, 2003.

GLEWWE, P.; JACOBY, H. G.; KING, E. M. Early childhood nutrition and academic achievement: a longitudinal analysis. Journal of Public Economics, v. 81, n. 3, p. 345-368, 2001.

GOLUB, M. S. et al. Activity and attention in zinc deprived adolescent monkeys. The American Journal of Clinical Nutrition, v. 64, n. 6, p. 905-915, 1996

GOLUB, M. S. et al. Developmental zinc deficiency and behavior. The Journal of Nutrition, v. $125, n$. 8 , p. 2263-2271, 1995.

GOLUB, M. S. et al. Food preference of young rhesus monkeys fed marginally zinc deficient diets. Primates, v. 32, n. 1, p. 49-59, 1991.

GOLUB, M. S. et al. Modulation of behavioral performance of prepubertal monkeys by moderate dietary zinc deprivation. The American Journal of Clinical Nutrition, v. 60, n. 2, p. 238-243, 1994.

GOLUB, M. S. et al. Studies of marginal zinc deprivation in rhesus monkeys. IV. Growth of infants in the first year. The American Journal of Clinical Nutrition, v. 40, n. 6, p. 1192-1202, 1984.

GOLUB, M. S. et al. Studies of marginal zinc deprivation in rhesus monkeys. VIII. Effects in early adolescence. The American Journal of Clinical Nutrition, v. 47, n. 6, p. 1046-1051, 1988.

GÓMEZ-PINILLA, F. Brain foods: the effects of nutrients on brain function. Nature Reviews Neuroscience, v. 9, n. 7, p. 568-578, 2008.

GRANHOLM, A. C. et al. Effects of a saturated fat and high cholesterol diet on memory and hippocampal morphology in the middle-aged rat. Journal of Alzheimer's Disease, v. 14, n. 2, p. 133-145, 2008. 
HAN, M., KIM, J. Effect of Dietary Iron Loading on Recognition Memory in Growing Rats. Ed. Brian Christie. PLOS ONE, v. 10, n. 3, 2015.

IBGE. Pesquisa de Orçamentos Familiares (POF) 2002-2003: antropometria e análise do estado nutricional de crianças e adolescentes no Brasil. Rio de Janeiro: IBGE; 2006.

INSTITUTE OF MEDICINE. Dietary reference intakes: the essential guide to nutriente requirements. Washington (DC): National Academy Press, 2006.

INSTITUTE OF MEDICINE. Dietary reference intakes for energy, carbohydrate, fiber, fat, fatty acids, cholesterol, protein, and amino acids. Washington (DC): National Academy Press, 2005.

INSTITUTE OF MEDICINE. Dietary reference intakes for vitamin A, vitamin K, arsenic, boron, chromium, copper, iodine, iron, manganese, molybdenum, nickel, silicon, vanadium, and zinc. Washington (DC): National Academy Press, 2002.

INSTITUTE OF MEDICINE. Dietary reference intakes for water, potassium, sodium, chloride, and sulfate. Washington (DC): National Academy Press, 2004.

LEAL, G. V. S. et al. Consumo alimentar e padrão de refeições de adolescentes, São Paulo, Brasil. Revista Brasileira de Epidemiologia, v. 13, n. 3, p. 457-467, 2010.

MAHAN, L. K.; ESCOTT-STUMP, S.; RAYMOND, J. L. Krause: alimentos, nutrição e dietoterapia. 13. ed. Rio de Janeiro: Elsevier, 2013. 1228 p.

MUÑOZ, P. et al. Iron Mediates N-Methyl-D-Aspartate Receptor-Dependent Stimulation of Calcium-Induced Pathways and Hippocampal Synaptic Plasticity. The Journal of Biological Chemistry, v. 286, n. 15, 2011.

NETO, A. S. et al. Estado nutricional e consumo alimentar de adolescentes da rede pública de ensino da cidade de São Mateus do Sul, Paraná, Brasil. Revista Brasileira de Saúde Materno Infantil, Recife, v. 8, n. 4, p. 435-443, 2008.

PONTES, R. et al. Influência da merenda escolar no estado nutricional. Cadernos da Escola de Saúde, Curitiba, v. 1, n 4, p. 64-77, 2010.

PRIORE, S. E. Nutrição e saúde na adolescência. PRIORE, Silvia Eloiza. Rio de Janeiro: Rubio, 2010. 460 p.

REZENDE, G. et al. Associação entre número de refeições diárias e excesso de peso em adolescentes. Nutrire, v. 34, n. Suplemento, p. 90-90, 2009.

SANTOS, J. S. et al. Perfil antropométrico e consumo alimentar de adolescentes de Teixeira de FreitasBahia. Revista de Nutrição, v. 18, n. 5, p. 623-632, 2005. 